\title{
Non-perturbative decay of non-Abelian hair
}

\section{Pablo A. Cano and Tomás Ortín}

Instituto de Física Teórica UAM/CSIC, C/ Nicolás Cabrera, 13-15, C.U. Cantoblanco, E-28049 Madrid, Spain

E-mail: pablo.cano@uam.es, Tomas.Ortin@csic.es

ABSTRACT: We construct a solution of Heterotic supergravity which interpolates between two different $\mathrm{AdS}_{3} \times \mathrm{S}^{3} \times \mathrm{T}^{4}$ geometries corresponding to the near-horizon limits of two 5-dimensional black holes, only one of which has non-Abelian hair. This solution can be used to estimate the amplitude of probability of the non-perturbative decay of the gauge 5 -brane responsible for the non-Abelian hair into eight solitonic 5-branes by evaluating its Euclidean action. The Wick rotation of this solution poses several problems which we argue can be overcome by using a non-extremal off-shell (NEOS) deformation of the solution. This NEOS field configuration can be Wick rotated straight away and its Euclidean action can be computed for any value of the deformation parameter. The Euclidean result can then be anti-Wick-rotated and its extremal limit gives the Euclidean action of the original solution, which turns out to be one half of the difference between the entropies of the 5 -dimensional black holes.

KeYwords: Black Holes in String Theory, Black Holes, p-branes, Superstring Vacua

ARXIV EPRINT: 1710.05052 


\section{Contents}

1 Introduction 1

2 Black holes with non-Abelian hair 3

3 Tunneling amplitude from the Euclidean path integral 6

$\begin{array}{ll}3.1 \text { Setting up the calculation } & 6\end{array}$

3.2 Dual action and solution 8

3.3 The NEOS deformation and its Euclideanization 9

3.4 Computation of the Euclidean action 11

4 Discussion $\quad 13$

\section{Introduction}

During the last few years, a number of solutions of 4- and 5-dimensional Super-EinsteinYang-Mills theories ${ }^{1}$ describing extremal black holes, strings and rings with different kinds of non-Abelian hair have been obtained in completely analytic form [1-7]. The naive form of their masses and entropies is puzzling, since the non-Abelian hair falls too fast at infinity to contribute to the mass but it is relevant at the horizon and contributes to the entropy. Thus, there seemed to be an infinite number of black holes with the same conserved charges at infinity but completely different entropies.

In order to clarify the situation, the embedding of a specially simple 5-dimensional black hole with non-Abelian hair in 10-dimensional Heterotic Supergravity was recently studied in ref. [8]. This embedding leads to the identification of the physical parameters of the 5-dimensional solution (Abelian charges and moduli) with the numbers of certain branes of Heterotic String Theory, namely fundamental strings (F1s), solitonic 5-branes (S5) and wave momentum in a compact direction (W). Furthermore, it was found that a single gauge 5-brane [9] is responsible for all the black hole's non-Abelian hair [10] and that this gauge 5-brane contributes to the same 5-dimensional charge as the S5-branes with 8 units. Thus, this 5-dimensional charge, which occurs in the mass formula, should be split into two different charges, Abelian and non-Abelian, both of which contribute to the mass. In this way, all the branes of the solution contribute to the mass, as expected, and the non-Abelian hair puzzle is solved by the correct stringy identification of the charges.

The solution to this puzzle poses new questions. Many of the non-Abelian (single) black hole and string solutions we have constructed have very interesting near-horizon geometries of a new kind that we have called dumbbell solutions in ref. [11] because they interpolate between two Bertotti-Robinson-like spaces $\operatorname{AdS}_{n} \times \mathrm{S}^{m}[12,13]$ with different

\footnotetext{
${ }^{1}$ These are the minimal supersymmetrizations of the Einstein-Yang-Mills theories that admit supersymmetric black-hole solutions. Therefore, they are gauged supergravities with non-Abelian gauge groups and, due to the last condition, they must have at least 8 supercharges.
} 
radii. They were first noticed in ref. [14], but they arise for several values of $n$ and $m$ in near-horizon limits of non-Abelian black holes and black strings. In particular, the nearhorizon geometry of the simple non-Abelian 5-dimensional black hole studied in ref. [8] interpolates between two $\mathrm{AdS}_{2} \times \mathrm{S}^{3}$ geometries with different radii which are found in two different limits, $\rho \rightarrow 0, \infty$ of the radial coordinate. One of them contains the contribution of the non-Abelian hair (i.e. the contribution of the gauge 5-brane) while the other does not and it is just the $\mathrm{AdS}_{2} \times \mathrm{S}^{3}$ one would obtain as the near-horizon geometry of a 3-charge Abelian black hole.

The existence of this solution suggests its potential use to study the quantum transition between one $\mathrm{AdS}_{2} \times \mathrm{S}^{3}$ vacuum and the other by Euclidean path integral methods, ${ }^{2}$ if a suitable instanton associated to this Lorentzian solution can be found. As a matter of fact, it is easier to work with and interpret the corresponding 10-dimensional solution of Heterotic Supergravity that one obtains by oxidizing the 5-dimensional dumbbell solution. In particular, as we are going to argue, in 10-dimensional language, the transition between the two vacua can be interpreted as a transition between a configuration that includes a gauge 5-brane and another in which there is no gauge 5-brane but there are 8 additional S5-branes or, in other words, the decay of a gauge 5-brane into 8 S5-branes (whose overall charge is the same).

In spite of its simplicity (as compared to the 5-dimensional one), it is very difficult to Wick-rotate the 10-dimensional solution to obtain the instanton whose Euclidean action we need to evaluate. We are going to argue that the most serious difficulties stem from the extremality of the solution. Indeed, the direct evaluation of the Euclidean action of extremal black holes has well-known problems $[16,17]$ that do not arise when one deals with the non-extremal solutions and finds their physical quantities of interest (Hawking temperature and Bekenstein-Hawking entropy) taking then the extremal limit of these quantities.

Here we propose to use a non-extremal deformation of the solution which is not a solution: a non-extremal off-shell (NEOS) deformation since all one needs is that the NEOS configurations interpolate between the same vacua as the original solution (so they contribute to the path integral for the same process), that they can be Wick-rotated and that they have finite Euclidean action to take, afterwards, the extremal limit. There is no systematic prescription to construct the NEOS configuration, but we manage to construct a one-parameter family with just the right properties and we evaluate its Euclidean action finding a result that we interpret physically as the amplitude of probability of decay of a gauge 5-brane into 8 S5-branes in a background containing a number of other branes. The value of the Euclidean action turns out to be one half of the difference of the entropies of the non-Abelian and Abelian black holes with those branes.

This paper is organized as follows: in section 2 we describe the solutions we are going to work with. In section 3 we compute the above-mentioned amplitude, setting up the calculation in section 3.1, rewriting it in section 3.2 to make the Wick rotation easier, introducing the NEOS configuration in section 3.3 and computing its Euclidean action in section 3.4. In section 4 we discuss our results.

\footnotetext{
${ }^{2}$ See, for instance, the collection of reprints [15].
} 


\section{Black holes with non-Abelian hair}

In this work we are going to study solutions of 10-dimensional Heterotic Supergravity with just one set of $\mathrm{SU}(2)$ gauge fields. Its action, in the string frame, is given by

$$
S=\frac{g_{s}^{2}}{16 \pi G_{N}^{(10)}} \int d^{10} x \sqrt{|g|} e^{-2 \phi}\left[R-4(\partial \phi)^{2}+\frac{1}{2 \cdot 3 !} H^{2}-\alpha^{\prime} F^{A} F^{A}\right],
$$

where the 2- and 3-form field strengths $F^{A}$ and $H$ are defined as

$$
\begin{aligned}
F^{A} & =d A^{A}+\frac{1}{2} \epsilon^{A B C} A^{B} \wedge A^{C} \\
H & =d B+2 \alpha^{\prime}\left(F^{A} \wedge A^{A}-\frac{1}{3 !} \epsilon^{A B C} A^{A} \wedge A^{B} \wedge A^{C}\right),
\end{aligned}
$$

$\alpha^{\prime}=l_{s}^{2}$ where $l_{s}$ is the string length and the 10 -dimensional Newton constant $G_{N}^{(10)}$ is given in terms of this string length and the string coupling constant $g_{s}$ by

$$
G_{N}^{(10)}=8 \pi^{6} g_{s}^{2} l_{s}^{8}
$$

The string coupling constant $g_{s}$ is equal to the vacuum expectation value of the exponential of the dilaton $g_{s}=\left\langle e^{\phi}\right\rangle$. In asymptotically-flat solutions, this should also be the value of the dilaton at infinity, and, therefore, in these solutions $g_{s}=e^{\phi_{\infty}}$.

This action is part of the low-energy effective field theory action of any of the two Heterotic Superstrings at first order in $\alpha^{\prime}$ since $\mathrm{SU}(2)$ is contained in both of their gauge groups. From the supersymmetry point of view, this action is complete, i.e. the bosonic part of a complete locally supersymmetric action. There is, however, another term which enters the action at first order in $\alpha^{\prime}$, proportional to $R_{-}^{2}$ where $R_{-}$is the Lorentz curvature 2 -form of one of the torsionful spin connections $\Omega_{ \pm \mu}^{a b}=\omega_{\mu}^{a b} \pm \frac{1}{2} H_{\mu}^{a b}$. Also the Bianchi identity of the 3 -form field strength $H$ has another term to first order in $\alpha^{\prime}$, proportional to $\operatorname{Tr}\left(R_{-} \wedge R_{-}\right)$. Introducing these terms alone would break the supersymmetric completeness of the action (a quartic term would be required to restore it [18]) and this is the reason why we are not including them because we rely on supersymmetric solution-generating techniques to obtain solutions. However, in order to consider the solutions of this action as legitimate solutions of the full Heterotic String effective action to first order in $\alpha^{\prime}$ expansion, one has to show that the terms quadratic in the curvature, evaluated on the solutions, are much smaller than those we have kept. In the solutions that we are going to consider (eq. (2.10)) the $\operatorname{Tr}\left(R_{-} \wedge R_{-}\right)$and other $R_{-}^{2}$ terms are of higher order in $\alpha^{\prime} .^{3}$

\footnotetext{
${ }^{3}$ From the point of view of $\alpha^{\prime}$ corrections, the vector fields in the Heterotic action may be used to suppress the terms coming from the torsionful spin connection. In our case, the torsionful spin connection associated to the S5-brane equals the SU(2) connection of the BPST instanton, so it is natural to use non-Abelian fields. However, also Abelian bundles at large charge have been used in the literature in order to suppress the $R_{-}^{2}$ terms [19, 20].
} 
In ref. [8] we obtained the following solution of SU(2) Heterotic Supergravity:

$$
\begin{aligned}
d s^{2} & =\frac{2}{Z_{-}} d u\left(d v-\frac{Z_{+}}{2} d u\right)-\tilde{Z}_{0}\left[d \rho^{2}+\rho^{2} d \Omega_{(3)}^{2}\right]-d y^{i} d y^{i}, \\
e^{2 \phi} & =e^{2 \phi_{\infty}} \frac{\tilde{Z}_{0}}{Z_{-}} \\
B & =-\frac{1}{Z_{-}} d v \wedge d u-\frac{\tilde{Q}_{0}}{4} \cos \theta d \psi \wedge d \varphi \\
A^{A} & =-\frac{\rho^{2}}{\kappa^{2}+\rho^{2}} v_{L}^{A}
\end{aligned}
$$

where $d \Omega_{(3)}^{2}$ is the metric of the unit, round $S^{3}, v_{L}^{A}$ are the 3 left-invariant Maurer-Cartan 1-forms of $\mathrm{SU}(2)$, the coordinates $y^{i}, i=6,7,8,9$ parametrize a $T^{4}$ and the $Z$ functions are given by

$$
\tilde{Z}_{0}=1+\frac{\tilde{Q}_{0}}{\rho^{2}}+8 \alpha^{\prime} \frac{\rho^{2}+2 \kappa^{2}}{\left(\kappa^{2}+\rho^{2}\right)^{2}}, \quad Z_{ \pm}=1+\frac{Q_{ \pm}}{\rho^{2}},
$$

where, in their turn, $\kappa$ is the size parameter of a SU(2) BPST instanton and the charges $\tilde{Q}_{0}, Q_{-}$and $Q_{+}$are related, respectively, to the number of solitonic five-branes, $N_{S 5}$, the number of fundamental strings, $N_{F 1}$, and the number of units of momentum flowing along the compact direction $u$ of radius $R_{z}, N_{W}$, by

$$
\tilde{Q}_{0}=l_{s}^{2} N_{S 5}, \quad Q_{-}=l_{s}^{2} g_{s}^{2} N_{F 1}, \quad Q_{+}=\frac{l_{s}^{4} g_{s}^{2}}{R_{z}^{2}} N_{W}
$$

Apart from the $S 5, F 1$ and $W$ ("Abelian") constituents, there is a single gauge 5-brane $N_{G 5}=1$ sourced by the $\mathrm{SU}(2)$ instanton [9].

When compactified on $T^{4} \times S^{1}$, this solution describes a five-dimensional black hole with non-Abelian hair, whose entropy and mass read

$$
\begin{aligned}
S & =2 \pi \sqrt{N_{S 5} N_{F 1} N_{W}}, \\
M & =\frac{R_{z}^{2}}{l_{s}^{2} g_{s}^{2}}\left(N_{S 5}+8 N_{G 5}\right)+\frac{R_{z}}{l_{s}^{2}} N_{F 1}+\frac{1}{R_{z}} N_{W} .
\end{aligned}
$$

The objects we have referred to as "Abelian" source Abelian 1-forms in the 5 -dimensional theory and their charges contribute both to the mass and entropy. The non-Abelian gauge 5-brane manifests itself as a globally regular gravitating instanton [10] which contributes to the mass as 8 solitonic 5-branes would, but does not contribute to the entropy at all. This makes this solution less thermodynamically favored than another one with $N_{S 5}^{\prime}=N_{S 5}+8$ solitonic 5-branes and no gauge 5-branes, which would have exactly the same mass, the same Abelian charges and moduli at infinity but larger entropy $S^{\prime}=2 \pi \sqrt{\left(N_{S 5}+8\right) N_{F 1} N_{W}}>S$, suggesting that the spontaneous decay of a gauge 5-brane into 8 solitonic 5 -branes is thermodynamically possible.

However, the decay of a gauge 5-brane into 8 solitonic 5-branes can never take place perturbatively, as the $\mathrm{SU}(2)$ instanton is protected by topology and it can only occur non-perturbatively, by quantum tunneling. 
In order to study this decay, it is convenient to consider a related solution, obtained by removing the 1's from the functions $Z_{0 \pm}$, which can be seen as the near-horizon limit of the above solution. This solution reads explicitly

$$
\begin{aligned}
d s^{2} & =\frac{2 \rho^{2}}{Q_{-}} d u d v-\frac{Q_{+}}{Q_{-}} d u^{2}-R^{2}\left(\frac{d \rho^{2}}{\rho^{2}}+d \Omega_{(3)}^{2}\right)-d y^{i} d y^{i}, \\
e^{2 \phi} & =e^{2 \phi_{\infty}} \frac{R^{2}}{Q_{-}} \\
B & =-\frac{\rho^{2}}{Q_{-}} d v \wedge d u-\frac{\tilde{Q}_{0}}{4} \cos \theta d \psi \wedge d \varphi \\
A^{A} & =-\frac{\rho^{2}}{\kappa^{2}+\rho^{2}} v_{L}^{A}
\end{aligned}
$$

where $R^{2}$ is the function

$$
R^{2}=\tilde{Q}_{0}+8 \alpha^{\prime} \frac{\rho^{2}\left(\rho^{2}+2 \kappa^{2}\right)}{\left(\kappa^{2}+\rho^{2}\right)^{2}} .
$$

In the absence of non-Abelian fields, this solution would just be $\mathrm{AdS}_{3} \times \mathrm{S}^{3} \times \mathrm{T}^{4}$, globally. This is the near-horizon geometry of the S5-F1-W brane configuration. However, the above solution, with the non-Abelian fields switched on, interpolates between two $\mathrm{AdS}_{3} \times \mathrm{S}^{3} \times \mathrm{T}^{4}$ geometries of different radii: ${ }^{4}$

- In the $\rho \rightarrow 0$ limit the squared radius of the $\mathrm{AdS}_{3} \times \mathrm{S}^{3}$ factor ${ }^{5}$ is $R_{0}^{2}=\tilde{Q}_{0}$.

- In the $\rho \rightarrow \infty$ limit the squared radius is $R_{\infty}^{2}=\tilde{Q}_{0}+8 \alpha^{\prime}$.

Furthermore, the gauge fields are also different in these two limits:

- In the $\rho \rightarrow 0$ limit $A_{0}^{A}=0$.

- In the $\rho \rightarrow \infty$ limit $A_{\infty}^{A}=-v_{L}^{A}$, which is a pure gauge configuration.

In order to compare the two limits, we must gauge-transform $A_{\infty}^{A}$ so that it also vanishes identically, $A_{\infty}^{A \prime}=0$. After this gauge transformation, the 2-form $B$, which transforms simultaneously via Nicolai-Townsend transformations due to the presence of the ChernSimons 3-form, takes the form

$$
B^{\prime}=-\frac{\rho^{2}}{Q_{-}} d v \wedge d u-\frac{\tilde{Q}_{0}+8 \alpha^{\prime}}{4} \cos \theta d \psi \wedge d \varphi,
$$

which, on account of the first of eqs. (2.7), tells us that the asymptotic geometry contains $N_{S 5}^{\prime}=N_{S 5}+8$ S5-branes.

${ }^{4}$ The metric of the $\mathrm{AdS}_{3}$ factor appears in a somewhat unconventional form

$$
d s_{\mathrm{AdS}_{3}}^{2}=\frac{2 \rho^{2}}{Q_{-}} d u d v-\frac{Q_{+}}{Q_{-}} d u^{2}-R_{0, \infty}^{2} \frac{d \rho^{2}}{\rho^{2}},
$$

but it can be checked that the Riemann curvature tensor corresponds to an $\mathrm{AdS}_{3}$ space of radius $R_{0, \infty}$.

${ }^{5}$ The radius of the $\mathrm{AdS}_{3}$ and $\mathrm{S}^{3}$ factors are equal, and we refer to this common value as the radius of the product geometry. 
We conclude that the complete solution eq. (2.10) can be interpreted as an interpolation between the near-horizon geometries of a configuration with $N_{S 5}$ S5-branes and $N_{G 5}=1$ gauge 5-brane and another configuration with $N_{S 5}^{\prime}=N_{S 5}+8$ S5-branes and $N_{G 5}=0$ gauge 5-branes.

\section{Tunneling amplitude from the Euclidean path integral}

\subsection{Setting up the calculation}

According to the Euclidean path integral approach, given an initial and a final state at fixed Euclidean times, the transition probability amplitude between them is given by

$$
\mathcal{Z}=\int \mathcal{D}[\Psi] e^{-S_{E}[\Psi]}
$$

where is the integral is taken over all the Euclidean field configurations $\Psi$ which satisfy the boundary conditions associated to the given initial and final states and $S_{E}[\Psi]$ is their Euclidean action. This probability can be well approximated by

$$
\mathcal{Z} \sim e^{-S_{E}\left[\Psi_{0}\right]}
$$

for a classical solution $\Psi_{0}$ with the given boundary conditions and finite Euclidean action, i.e. an instanton. In some cases (when the initial and final states are vacua) this probability can be interpreted as the decay rate of a metastable vacuum into a more stable one.

The simplest prescription to obtain a Euclidean solution is to Wick-rotate $(t=-i \tau)$ a Lorentzian one. However, when applied to non-trivial field configurations (non-static metrics, for example) this naive prescription fails to give real solutions of Euclidean signature unless some parameters of the solution are analytically continued into the complex domain (see, for instance, the seminal ref. [21]). These Euclidean solutions can be thought of as real sections of a complexified solution obtained by analytical continuation, but their existence is by no means guaranteed and, in general, finding a real solution of Euclidean signature associated to a Lorentzian one is a well-known and complicated problem. ${ }^{6}$

Here, we would like to find a real Euclidean solution associated to the Lorentzian dumbbell solution described in eqs. (2.10) of the previous section. Such a solution, if of finite Euclidean action, could be interpreted as an instanton interpolating between the two vacua $N_{S 5}, N_{G 5}=1, N_{F 1}, N_{W}$ and $N_{S 5}+8, N_{G 5}=0, N_{F 1}, N_{W}$ and the (minus) exponential of its Euclidean action would give the probability of decay from one vacuum to the other. Predictably, after the preceeding discussion, in the search for this real Euclidean solution we are going to meet several problems that we are going to try to solve.

The first problem arises in the Wick rotation of the Kalb-Ramond 2-form $B$, which makes the electric part purely imaginary. This is usually dealt with by Wick-rotating the "electric charge," $\left(Q_{-}\right.$, here) as well, but this would prove fatal in this case because it would make the dilaton and several components of the metric imaginary.

\footnotetext{
${ }^{6}$ See e.g. ref. [22] and references therein.
} 
As we are going to explain, the root of this problem may lie in the extremality of our solution. Let us compare the Wick rotation of the solution at hands with that of a more familiar solution: an electrically-charged Reissner-Norström black hole.

In the non-extremal regime there is no problem with the simultaneous Wick rotation of the time $t=-i \tau$ and the electric charge $q=-i q_{E}$ because in the standard coordinates in which the metric takes the form

$$
d s^{2}=\frac{\left(r-r_{+}\right)\left(r-r_{-}\right)}{r^{2}} d t^{2}-\frac{r^{2}}{\left(r-r_{+}\right)\left(r-r_{-}\right)} d r^{2}-r^{2} d \Omega_{(2)}^{2},
$$

with $r_{ \pm}=M \pm \sqrt{M^{2}-Q^{2}}$, the electric charge $Q$ only occurs quadratically in the metric. There is no extremal limit of this Euclidean solution, though, as the Lorentzian extremality condition $M^{2}-Q^{2}=0$ becomes $M^{2}+Q_{E}^{2}=0$ and the near-horizon limit is always $\mathbb{E}^{2} \times S^{2}$ (corresponding to the Lorentzian Rindler $\times S^{2}$ ) and not $\mathbb{H}^{2} \times S^{2}$ (which would correspond to $\mathrm{AdS}_{2} \times \mathrm{S}^{2}$ ). Of course, one can always take the extremal limit of the physical quantities computed in the non-extremal case after they are re-expressed in terms of the Lorentzian charges. This is how, typically, the entropy and (vanishing) temperature of extremal black holes are computed in the Euclidean approach because the direct Wick rotation and the computation of the Euclidean action of the extremal solution present very serious problems. ${ }^{7}$

Let us start with the problems presented by the direct Wick rotation of the extremal solution.

First of all, if one tries to Wick-rotate directly the extremal Lorentzian solution in which $r_{+}=r_{-}=M= \pm Q$ one finds that one has to Wick-rotate the mass as well, losing the reality of the metric. In our case there seems to be no way to make all the Wick-rotated fields real (specially the metric, due to its complicated form) simultaneously no matter how we treat the parameters of the solution.

There is a way out in the context of the 4-dimensional Maxwell-Einstein theory: one can dualize the electric charge into a magnetic charge, which needs not be Wick-rotated. Dyonic solutions such as eqs. (2.10) can be more difficult to rotate into a purely magnetic solution but we can split the 2-form into its electric and a magnetic parts and dualize only the electric one obtaining two magnetic fields (a 2-form and a 6 -form) which do not need to be Wick-rotated. We will explain how to do this in detail later but we can advance that this trick turns out to be only good enough to keep the solution real for $Q_{+}=0$. This strongly suggests that we should try to work with a non-extremal Euclidean solution and then take the extremal limit of the Lorentzian results.

On top of the problems related to the Wick rotation there is another problem that seems to affect extremal solutions only and which supports the need of working with non-extremal solutions. As shown in refs. [16, 17], a direct calculation of the entropy of the extremal Reissner-Nordström black hole within the Euclidean approach gives a re-

\footnotetext{
${ }^{7}$ The Wick rotation of the extremal Kerr black hole provides another, slightly different, example of the same problem which can only be solved by working with non-extremal Kerr black holes, which can be Wick-rotated consistently if one also rotates the angular momentum, and then taking the extremal limit of the results expressed in terms of the Lorentzian variables.
} 
sult $\left(S_{\mathrm{BH}}=0\right)$ which differs from the extremal limit of the entropy of the non-extremal black holes, which is the same as the value obtained by counting microstates in the String Theory context [23-25]. The technical reason is the existence of an inner boundary in the extremal Euclidean solution which does not exist in the non-extremal family of solutions for any value of the physical parameters.

The need to work with a non-extremal solution raises another problem, because the non-extremal version of the black-hole solution eqs. (2.5) is not known and it has been argued that it may not exist. We are going to circumvent this problem by constructing a 1-parameter non-extremal deformation of the solution eqs. (2.10) which is not a solution of the equations of motion but interpolates between the same two vacua as the extremal solution. This non-extremal off-shell (NEOS) deformation can be understood as a mere regularization procedure or as a computation of the action over an off-shell family of field configurations that contribute to the path integral in eq. (3.1) because they have the boundary conditions demanded in this case. The extremal limit is, at the same time an extremum of the action because it is a solution of the classical equations of motion and, clearly, it makes sense to compute the action over the complete family of configurations and then take the extremal limit.

In the rest of this section we are going to carry out the program explained above. First of all, we are going to dualize the electric part of the Kalb-Ramond 2-form into a magnetic 6 -form. This has to be done in the action and in the solution simultaneously. Next, we will make a first attempt at the Wick rotation and we will see that for $Q_{+} \neq 0$ we need the NEOS deformation. Finally, we will compute the Euclidean action for this family of field configurations, taking into account all the boundary terms.

\subsection{Dual action and solution}

In order to dualize the electric part of the Kalb-Ramond 2-form $B$ we replace it by the sum of a pair of 2-forms $B_{1}+B_{2}$ such that $H_{1} \cdot H_{2}=0$ and then dualize the second into a 6-form $\tilde{B}_{2}$ with 7 -form field strength $\tilde{H}_{2}=\star e^{-2 \phi} H_{2}$ such that $H_{1} \wedge \tilde{H}_{2}=0$. The resulting action is ${ }^{8}$

$$
\begin{aligned}
S= & \frac{g_{s}^{2}}{16 \pi G_{N}^{(10)}} \int d^{10} x \sqrt{|g|}\left\{e^{-2 \phi}\left[R-4(\partial \phi)^{2}+\frac{1}{2 \cdot 3 !} H^{2}-\alpha^{\prime} F^{A} F^{A}\right]\right. \\
& \left.+\frac{e^{2 \phi}}{2 \cdot 7 !} \tilde{H}^{2}-\frac{\alpha^{\prime}}{2 \cdot 6 ! \sqrt{|g|}} \epsilon^{\mu_{1} \cdots \mu_{6} \alpha \beta \gamma \delta} \tilde{B}_{\mu_{1} \cdots \mu_{6}} F_{\alpha \beta}^{A} F_{\gamma \delta}^{A}\right\},
\end{aligned}
$$

and any solution of this action satisfying the constraint $H \wedge \tilde{H}=0$ is a solution of the original Heterotic Supergravity with

$$
H=H+e^{2 \phi} \star \tilde{H} .
$$

\footnotetext{
${ }^{8}$ In the process of dualization a boundary term is also generated, which is not shown here because it does not change the equations of motion, but which will be taken into account in the computation of the Euclidean action.
} 
We can reexpress the solution eqs. (2.10) as the following purely magnetic solution of the above action whose Wick rotation is potentially simpler:

$$
\begin{aligned}
d s^{2} & =\frac{2 \rho^{2}}{Q_{-}} d u d v-\frac{Q_{+}}{Q_{-}} d u^{2}-R^{2}\left(\frac{d \rho^{2}}{\rho^{2}}+d \Omega_{(3)}^{2}\right)-d y^{i} d y^{i}, \\
e^{2 \phi} & =e^{2 \phi_{\infty}} \frac{R^{2}}{Q_{-}} \\
B & =-\frac{\tilde{Q}_{0}}{4} \cos \theta d \psi \wedge d \varphi \\
\tilde{B} & =-\frac{e^{-2 \phi_{\infty}} Q_{-}}{4} \cos \theta d \psi \wedge d \varphi \wedge d y^{6} \wedge d y^{7} \wedge d y^{8} \wedge d y^{9}, \\
A^{A} & =-\frac{\rho^{2}}{\kappa^{2}+\rho^{2}} v_{L}^{A} .
\end{aligned}
$$

In order to proceed with Wick rotation, we first need to identify an appropriate time coordinate. Let us first begin with the case in which there is no momentum, $Q_{+}=0$. In this case, we can make the change of variables $v=(t-x) / \sqrt{2}, u=(t+x) / \sqrt{2}$, which makes $2 d u d v=d t^{2}-d x^{2}$. Then we substitute $t=-i \tau$, and redefine with a global sign the metric to get a positive-definite Euclidean metric (the rest of fields are unaffected)

$$
d s_{E}^{2}=\frac{\rho^{2}}{Q_{-}}\left(d \tau^{2}+d x^{2}\right)+R^{2}\left(\frac{d \rho^{2}}{\rho^{2}}+d \Omega_{(3)}^{2}\right)+d y^{i} d y^{i} .
$$

This solution interpolates between two $\mathbb{H}^{3} \times \mathrm{S}^{3}$ geometries of radii $R_{0}$ and $R_{\infty}$ and is a gravitational instanton which represents a tunneling history in which one $\mathbb{H}^{3} \times S^{3}$ vacuum decays into another one of larger radius, or, according to the previous discussion, a history in which a gauge 5 -brane decays into 8 solitonic 5 -branes. We may just compute the Euclidean action for this solution which is real but, as we will see, it vanishes.

\subsection{The NEOS deformation and its Euclideanization}

This is closely related to the fact that the solution with $Q_{+}=0$ does not produce a black hole in five dimensions. Indeed, it is known that the on-shell Euclidean action for black hole solutions is related to the entropy of the black hole. For $Q_{+}=0$ the entropy of the would-be black hole vanishes so it is reasonable that the Euclidean action does so. It is necessary to have $Q_{+} \neq 0$ in order to get a non-vanishing action. ${ }^{9}$

When $Q_{+} \neq 0$ things are more involved: after the change of variables $v=(t-x) / \sqrt{2}$, $u=(t+x) / \sqrt{2}$

$$
d s^{2}=\frac{1}{Q_{-}}\left[\left(\rho^{2}-\frac{Q_{+}}{2}\right) d t^{2}-Q_{+} d t d x-\left(\rho^{2}+\frac{Q_{+}}{2}\right) d x^{2}\right]-R^{2}\left(\frac{d \rho^{2}}{\rho^{2}}+d \Omega_{(3)}^{2}\right)-d y^{i} d y^{i}
$$

there is a crossed term in the metric $Q_{+} d t d x$ that becomes imaginary after the Wick rotation unless we rotate $Q_{+}$as well. But $Q_{+}$occurs in more places in the metric, which would become complex.

\footnotetext{
${ }^{9}$ The $Q_{+}=0$ solution becomes singular in $d=5$, while, for $Q_{+} \neq 0$ it is $\operatorname{AdS}_{2} \times \mathrm{S}^{3}$, the near-horizon limit of a regular, extremal black hole.
} 
As explained before, this problem can be solved by using a 1-parameter a NEOS deformation of the metric ${ }^{10}$

$$
\begin{aligned}
d s_{\mathrm{NEOS}}^{2}= & \frac{1}{Q_{-}}\left[\left(\left(\rho+\rho_{0}\right)^{2}-\frac{Q_{+}}{2}\right) d t^{2}-a d t d x-\left(\left(\rho+\rho_{0}\right)^{2}+\frac{Q_{+}}{2}\right) d x^{2}\right] \\
& -R^{2}\left[\frac{\left(\rho+\rho_{0}\right)^{2} d \rho^{2}}{\left(\rho+\rho_{0}\right)^{4}-\rho_{0}^{4}}+d \Omega_{(3)}^{2}\right]-d y^{i} d y^{i},
\end{aligned}
$$

where

$$
\rho_{0}^{2} \equiv \frac{1}{2} \sqrt{Q_{+}^{2}-a^{2}}
$$

and $R(\rho)$ has the same form as before.

Even though the NEOS configuration (3.9) is not a solution in general, it shares with the original extremal solution several interesting properties. If we consider the case of pure gauge vector fields, so that $R(\rho)$ is constant, it is a solution with the geometry $\mathrm{AdS}_{3} \times S^{3} \times \mathrm{T}^{4}$, albeit in different coordinates. With non-trivial gauge fields the metric interpolates between two $\mathrm{AdS}_{3} \times \mathrm{S}^{3} \times \mathrm{T}^{4}$ geometries with radii $R_{0}$ and $R_{\infty}$, so the NEOS configurations contribute to the path integral that describes the transition between these two vacua, and the $a=Q_{+}$extremizes the Euclidean action.

Furthermore, for arbitrary values of $a$, this metric can be analytically continued to a Euclidean metric by making $t=-i \tau, a=i \aleph$. In general, it is not a solution, but when the "extremality condition" $a^{2}=Q_{+}^{2}$ is satisfied in the Lorentzian configuration, we recover the solution (3.8).

As discussed above, we are going to Wick-rotate the above NEOS configuration first, then we are going to compute its Euclidean action and at the end we are going to take the $a^{2} \rightarrow Q_{+}^{2}$ limit in the result.

First, let us massage a bit the metric by making the change of coordinates

$$
\begin{aligned}
& t=\frac{\sqrt{Q_{+} / 2+\rho_{0}^{2}}}{\sqrt{2} \rho_{0}} t^{\prime}-\frac{\sqrt{Q_{+} / 2-\rho_{0}^{2}}}{\sqrt{2} \rho_{0}} y, \\
& x=\frac{\sqrt{Q_{+} / 2+\rho_{0}^{2}}}{\sqrt{2} \rho_{0}} y-\frac{\sqrt{Q_{+} / 2-\rho_{0}^{2}}}{\sqrt{2} \rho_{0}} t^{\prime},
\end{aligned}
$$

which is a Lorentz boost. This sets the metric in a diagonal form

$$
\begin{aligned}
d s^{2}= & \frac{1}{Q_{-}}\left\{\left[\left(\rho+\rho_{0}\right)^{2}-\rho_{0}^{2}\right] d t^{\prime 2}-\left[\left(\rho+\rho_{0}\right)^{2}+\rho_{0}^{2}\right] d y^{2}\right\} \\
& -R^{2}\left[\frac{\left(\rho+\rho_{0}\right)^{2} d \rho^{2}}{\left(\rho+\rho_{0}\right)^{4}-\rho_{0}^{4}}+d \Omega_{(3)}^{2}\right]-d y^{i} d y^{i} .
\end{aligned}
$$

In the $\rho \rightarrow 0$ limit, the $t^{\prime}-\rho$ sector of the metric is just a $1+2$-dimensional Rindler spacetime and, therefore, it corresponds to a non-extremal horizon whose Hawking temperature we will compute later.

To complete the definition of this field configuration we have to determine the period of the coordinate $y$, which is assumed to be compact in the original solution we started

\footnotetext{
${ }^{10}$ The matter fields in the solution eqs. (3.6) are unaffected by this deformation.
} 
with. By comparing the area of the horizon of this metric and of the original one we conclude that ${ }^{11}$

$$
2 \pi R_{y} \equiv \int d y=\frac{\sqrt{Q_{+} / 2}}{\rho_{0}} \int d u=\frac{\sqrt{Q_{+} / 2}}{\rho_{0}} 2 \pi R_{z} .
$$

Then, we perform Wick rotation: $t^{\prime}=-i \tau, a=i \aleph$ and, after an overall change of sign, we get the Euclidean metric

$$
\begin{aligned}
d s_{\mathrm{ENEOS}}^{2}= & \frac{1}{Q_{-}}\left\{\left[\left(\rho+\rho_{0}\right)^{2}-\rho_{0}^{2}\right] d \tau^{2}+\left[\left(\rho+\rho_{0}\right)^{2}+\rho_{0}^{2}\right] d y^{2}\right\} \\
& +R^{2}\left[\frac{\left(\rho+\rho_{0}\right)^{2} d \rho^{2}}{\left(\rho+\rho_{0}\right)^{4}-\rho_{0}^{4}}+d \Omega_{(3)}^{2}\right]+d y^{i} d y^{i},
\end{aligned}
$$

where now

$$
\rho_{0}^{2}=\frac{1}{2}\left[Q_{+}^{2}+\aleph^{2}\right]^{1 / 2} .
$$

Note that the $R=$ constant configurations (with pure gauge vector fields) are solutions (just as their Lorentzian partners) and have the geometry of $\mathbb{H}^{3} \times \mathrm{S}^{3} \times \mathrm{T}^{4}$ with radius $R$.

In the $\rho \rightarrow 0$ limit, redefining the radial coordinate $\rho \equiv \rho_{0} r^{2} / R_{0}^{2}$, the metric takes the form

$$
d s_{E}^{2}=\frac{1}{Q_{-}}\left[2 r^{2} \frac{\rho_{0}^{2}}{R_{0}^{2}} d \tau^{2}+2 \rho_{0}^{2} d y^{2}\right]+d r^{2}+R_{0}^{2} d \Omega_{(3)}^{2}+d y^{i} d y^{i},
$$

and, to avoid a conical singularity at $r=0$, the Euclidean time $\tau$ must be periodic with period $\beta$ so that the Hawking temperature (undoing the Wick rotation of the $a$ parameter) is

$$
T_{H}=\beta^{-1}=\frac{1}{2 \pi} \sqrt{\frac{\left(Q_{+}^{2}-a^{2}\right)^{1 / 2}}{\tilde{Q}_{0} Q_{-}}},
$$

and vanishes in the $a \rightarrow Q_{+}$limit.

\subsection{Computation of the Euclidean action}

The complete Euclidean action that we want to compute is given by

$$
\begin{aligned}
S_{E}= & \frac{g_{s}^{2}}{16 \pi G_{N}^{(10)}} \int_{\mathcal{M}} d^{10} x \sqrt{\left|g_{E}\right|}\left\{e^{-2 \phi}\left[R-4(\partial \phi)^{2}+\frac{1}{2 \cdot 3 !} H^{2}+\alpha^{\prime} F^{A} F^{A}\right]\right. \\
& \left.+\frac{e^{2 \phi}}{2 \cdot 7 !} \tilde{H}^{2}+\alpha^{\prime} \frac{\epsilon^{\mu_{1} \cdots \mu_{6} \alpha \beta \gamma \delta}}{2 \cdot 6 ! \sqrt{\left|g_{E}\right|}} \tilde{B}_{\mu_{1} \cdots \mu_{6}} F^{A}{ }_{\alpha \beta} F^{A}{ }_{\gamma \delta}\right\} \\
& +\frac{g_{s}^{2}}{8 \pi G_{N}^{(10)}} \int_{\partial \mathcal{M}} d^{9} x \sqrt{\left|h_{E}\right|}\left[-\frac{e^{2 \phi}}{2 \cdot 6 !} n^{\mu}(\tilde{H} \cdot \tilde{B})_{\mu}-e^{-2 \phi} K\right]+c,
\end{aligned}
$$

where $K$ is the trace of the extrinsic curvature of the boundary $\partial \mathcal{M}, c$ is a normalization constant which is fixed by the criterium that $S_{E}=0$ when evaluated on the vacuum (taken to be $\mathbb{H}^{3} \times \mathrm{S}^{3} \times \mathrm{T}^{4}$ with radius $\left.R_{\infty}\right)$ and $(\tilde{H} \cdot \tilde{B})_{\mu} \equiv \tilde{H}_{\mu \nu_{1} \cdots \nu_{6}} \tilde{B}^{\nu_{1} \cdots \nu_{6}}$. This surface term

\footnotetext{
${ }^{11}$ Actually, we only need to impose that the period of $y$ tends to this quantity in the limit $a^{2} \rightarrow Q_{+}^{2}$.
} 
appears when we dualize the Kalb-Ramond 2-form $B$. For the normalization constant, we will use the usual prescription

$$
c=\frac{g_{s}^{2}}{8 \pi G_{N}^{(10)}} \int_{\partial \mathcal{M}} d^{9} x \sqrt{\left|h_{E}\right|} e^{-2 \phi} K_{0},
$$

where $K_{0}$ is the extrinsic curvature of the boundary when it is embedded in the vacuum.

In order to evaluate the integrand of the action it is convenient to use the equations of motion, but here we have to be very careful because we are not dealing with a solution, and not all of them are satisfied. In particular, the equation of motion of the dilaton is not satisfied and we have to add a "source" term $\delta(\rho ; \aleph)$

$$
e^{-2 \phi}\left[R-4(\partial \phi)^{2}+\frac{1}{2 \cdot 3 !} H^{2}+\alpha^{\prime} F^{A} F^{A}\right]-4 \nabla_{\mu}\left(e^{-2 \phi} \partial^{\mu} \phi\right)-\frac{e^{2 \phi}}{2 \cdot 7 !} \tilde{H}^{2}=\delta(\rho ; \aleph),
$$

which can be simply computed by plugging the fields in the l.h.s.

We know, however, that $\delta=0$ for global $\mathbb{H}^{3} \times \mathrm{S}^{3} \times \mathrm{T}^{4}$ and also that $\lim _{\rho \rightarrow \infty} \delta(\rho ; \aleph)=0$, because the configuration we are considering asymptotically tends to a solution. Indeed, $\delta$ decays so fast that the integral

$$
\Delta(\aleph) \equiv \frac{1}{Q_{-}} \int_{0}^{\infty} d \rho\left(\rho+\rho_{0}\right) R^{4}(\rho) \delta(\rho ; \aleph),
$$

converges.

Using the dilaton equation, the Euclidean action eq. (3.18) takes a simpler form:

$$
\begin{aligned}
S_{E}= & \frac{g_{s}^{2}}{16 \pi G_{N}^{(10)}} \int_{\mathcal{M}} d^{10} x \sqrt{\left|g_{E}\right|}\left\{\frac{e^{2 \phi}}{7 !} \tilde{H}^{2}+\alpha^{\prime} \frac{\epsilon^{\mu_{1} \cdots \mu_{6} \alpha \beta \gamma \delta}}{2 \cdot 6 ! \sqrt{\left|g_{E}\right|}} \tilde{B}_{\mu_{1} \cdots \mu_{6}} F_{\alpha \beta}^{A} F_{\gamma \delta}^{A}+\delta(\rho ; \aleph)\right\} \\
& +\frac{g_{s}^{2}}{8 \pi G_{N}^{(10)}} \int_{\partial \mathcal{M}} d^{9} x \sqrt{\left|h_{E}\right|}\left\{n_{\mu}\left[2 e^{-2 \phi} \partial^{\mu} \phi-\frac{e^{2 \phi}}{2 \cdot 6 !}(\tilde{H} \cdot \tilde{B})^{\mu}\right]-e^{-2 \phi}\left(K-K_{0}\right)\right\} .
\end{aligned}
$$

Next, we massage the first term in the integrand

$$
\frac{e^{2 \phi}}{7 !} \tilde{H}^{2}=\frac{e^{2 \phi}}{6 !} \tilde{H}^{\mu_{1} \cdots \mu_{7}} \nabla_{\mu_{1}} \tilde{B}_{\mu_{2} \cdots \mu_{7}}=\frac{1}{6 !} \nabla_{\mu}\left[e^{2 \phi}(\tilde{H} \cdot \tilde{B})^{\mu}\right]-\frac{1}{6 !} \nabla_{\rho}\left(e^{2 \phi} \tilde{H}^{\rho \mu_{1} \cdots \mu_{6}}\right) \tilde{B}_{\mu_{1} \cdots \mu_{6}} .
$$

The first term cancels identically the $\tilde{H} \cdot \tilde{B}$ boundary terms and the second combines with the $\tilde{B} F F$ term into a term proportional to the equation of motion of $\tilde{B},{ }^{12}$ which happens to be identically satisfied, ${ }^{13}$ so we get

$S_{E}(\aleph)=\frac{g_{s}^{2}}{16 \pi G_{N}^{(10)}}\left\{\int_{\mathcal{M}} d^{10} x \sqrt{\left|g_{E}\right|} \delta(\rho ; \aleph)+2 \int_{\partial \mathcal{M}} d^{9} x \sqrt{\left|h_{E}\right|} e^{-2 \phi}\left[2 n_{\mu} \partial^{\mu} \phi-\left(K-K_{0}\right)\right]\right\}$.

${ }^{12}$ This term has the form

$$
-\frac{1}{6 !} \tilde{B}_{\mu_{1} \cdots \mu_{6}}\left[\nabla_{\rho}\left(e^{2 \phi} \tilde{H}^{\rho \mu_{1} \cdots \mu_{6}}\right)-\alpha^{\prime} \frac{\epsilon^{\mu_{1} \cdots \mu_{6} \alpha \beta \gamma \delta}}{2 \sqrt{\left|g_{E}\right|}} F^{A}{ }_{\alpha \beta} F_{\gamma \delta}^{A}\right] .
$$

\footnotetext{
${ }^{13}$ Incidentally, this is the same result we would have obtained had we worked with a complex $B$, which shows that, in this case, the imaginary electric part is not harmful.
} 
Since the only non-compact coordinate is $\rho$, the boundary of $\mathcal{M}$ consists just of the asymptotic region $\rho=\rho_{\infty}$, where $\rho_{\infty}$ is a regulator that must be taken to infinity eventually. In the Euclidean NEOS configuration that we are considering, with the Euclidean time compactified with the period $\beta=1 / T_{H}$ the region $\rho=0$ is not a boundary.

In the limit $\rho \rightarrow \infty$

$$
n^{\mu} \partial_{\mu} \phi=\mathcal{O}\left(\rho^{-4}\right), \quad \text { and } \quad K-K_{0}=\mathcal{O}\left(\rho^{-4}\right),
$$

so these terms decay too fast to contribute to the integral. Therefore, only the bulk term gives a non-zero contribution and the Euclidean action is given by

$$
S_{E}(\aleph)=\frac{g_{s}^{2}}{16 \pi G_{N}^{(10)}} V_{\mathrm{T}^{4}} V_{\mathrm{S}^{3}} 2 \pi R_{y} \beta \Delta(\aleph),
$$

where the 10-dimensional Newton constant is given in eq. (2.4), $V_{\mathrm{T}^{4}}=\left(2 \pi l_{s}\right)^{4}$ is the volume of the $\mathrm{T}^{4}, V_{\mathrm{S}^{3}}=2 \pi^{2}$ is the volume of the $\mathrm{S}^{3}$ of unit radius, $R_{y}$ is the radius of the compact coordinate $y$ given in eq. (3.13), $\beta=1 / T_{H}$ is the period of the Euclidean time given implicitly in eq. (3.17) and $\Delta(\aleph)$ is the integral defined in eq. (3.21). Substituting in the above expression, we find that

$$
S_{E}(\aleph)=\frac{\pi R_{z} \sqrt{\tilde{Q}_{0} Q_{+} Q_{-}}}{2 l_{s}^{4}} \frac{\Delta(\aleph)}{\rho_{0}^{2}} .
$$

Note that this is a finite result for all the finite values of $\rho_{0}$. These NEOS configurations are, therefore, instantons, even if they are not solutions. We now have to undo the Wick rotation $\aleph=-i a$, so that $\rho_{0}^{2}=\frac{1}{2}\left(Q_{+}^{2}-a^{2}\right)^{1 / 2}$ and take the limit $a \rightarrow Q_{+}$.

In this limit both $\Delta(a)$ and $\rho_{0}$ go to zero, but the limit of $\Delta(a) / \rho_{0}^{2}$ turns out to be finite and takes the value

$$
\lim _{a \rightarrow Q_{+}} \frac{\Delta(a)}{\rho_{0}^{2}}=g_{s}^{2} \log \left(1+\frac{8 \alpha^{\prime}}{\tilde{Q}_{0}}\right)=g_{s}^{2} \log \left(1+\frac{8}{N_{S 5}}\right) \approx \frac{8 g_{S}^{2}}{N_{S 5}},
$$

where we have used the assumption that $N_{S 5} \gg 8$.

This leads us to our final result for the Euclidean action associated to the Lorentzian solution interpolating between two brane configurations we started from

$$
S_{E}=4 \pi \sqrt{\frac{N_{F 1} N_{W}}{N_{S 5}}},
$$

which leads to the transition probability

$$
|\mathcal{A}|^{2} \sim e^{-8 \pi \sqrt{\frac{N_{F 1} N_{W}}{N_{S 5}}}}
$$

\section{Discussion}

First of all, we observe that, quite remarkably, this result coincides with $e^{-\Delta S_{\mathrm{BH}}}$, where $\Delta S_{\mathrm{BH}}$ is the change of Bekenstein-Hawking entropy in the process:

$$
\Delta S_{\mathrm{BH}}=2 \pi \sqrt{N_{F 1} N_{W}\left(N_{S 5}+8\right)}-2 \pi \sqrt{N_{F 1} N_{W} N_{S 5}} \approx 8 \pi \sqrt{\frac{N_{F 1} N_{W}}{N_{S 5}}},
$$


which is what one can expect on general grounds: for a single black hole, the Euclidean action is proportional to the Bekenstein-Hawking entropy $[21,26]^{14}$ and the Lorentzian solution we started from was interpreted as connecting two different black-holes nearhorizon geometries, so it is natural that the Euclidean action yields the difference between the entropies of two black holes.

Notice that the sign in the exponent is opposite to what one would expect from statistical mechanics, where the decay rate would be estimated to be of the order of $e^{+\Delta S}$. In our case, the entropy difference is positive, so the process is favored thermodynamically. However, the decay process involves topology change, which is highly suppressed. This way we can interpret the minus sign in $e^{-\Delta S}$ as the fact that topology constrains the decay so effectively that it succeeds in suppressing the process even though it involves an increase of entropy.

Given the composition of the string background corresponding to the black hole (F1s, NS5s etc), the result can also be interpreted as the decay rate of a gauge 5-brane which lives in a configuration of fundamental strings, momentum waves and solitonic 5-branes.

When the numbers of each kind of component are comparable, this probability is tiny and the gauge 5-brane, though unstable, is long lived. However, when the number of S5branes is much larger than the number of strings and waves, $N_{S 5}>N_{F 1} N_{W}$, the gauge 5-branes decay quickly into S5-branes. One may say that S5-branes are "hungry" for gauge 5 -branes, and the larger their number the faster they will eat them.

The result suggests that the non-Abelian 5-dimensional black hole eq. (2.5) whose near-horizon limit gives the Lorentzian "dumbbell" solution we started from eq. (2.10) is non-perturbatively unstable. Furthermore, and possible related to this fact, it seems very difficult or, perhaps, it is impossible, to find non-extremal black holes with the same charges and non-Abelian fields. Clearly, more work is necessary to clarify the situation.

Finally, we should comment on the relation between the result obtained here and the method employed and Brill's work ref. [27] in which he computed the Euclidean action of an instanton whose Lorentzian counterpart connects several (at least three) asymptotic $\mathrm{AdS}_{2} \times \mathrm{S}^{2}$ geometries and which has non-trivial topology. ${ }^{15}$ These solutions can be used to study the non-perturbative splitting of a Reissner-Nordström black hole into smaller black holes. In that case the Wick rotation offers no special complications but the geometry of the instanton is very complicated and it is not clear how to deal with the inner boundaries identified in refs. $[16,17]$. The solution studied here is topologically simpler (at least from the metric point of view), but we have argued that a non-extremal off-shell (NEOS) deformation had to be used to go to the Euclidean, compute the action and then go back to Lorentzian signature and take the extremal limit, avoiding many of the pitfalls one finds along the way.

We expect the NEOS technique developed here to be of further use in other contexts.

\footnotetext{
${ }^{14}$ Again, we stress that, for extremal black holes, this calculation has to be made in the family of nonextremal black holes and then one has to take the extremal limit in the result to avoid the problems found in refs. [16, 17], as we have done here using a NEOS family.

${ }^{15}$ These solutions are obtained from the Papapetrou-Majumdar family of solutions that describe ReissnerNordström black holes in equilibrium [28, 29] with more than one center. Removing the constant part of the harmonic function one finds an $\mathrm{AdS}_{2} \times \mathrm{S}^{2}$ region in the near-horizon limits and another $\mathrm{AdS}_{2} \times \mathrm{S}^{2}$ region at infinity whose charges are the sum of all those of the other regions. With just one center, one finds a single, global $\mathrm{AdS}_{2} \times \mathrm{S}^{2}$ solution, with $N$ centers one find $N+1 \mathrm{AdS}_{2} \times \mathrm{S}^{2}$ regions.
} 


\section{Acknowledgments}

The authors would like to thank Patrick Meessen and Pedro F. Ramírez for many useful conversations. This work has been supported in part by the MINECO/FEDER, UE grant FPA2015-66793-P and by the Spanish Research Agency (Agencia Estatal de Investigación) through the grant IFT Centro de Excelencia Severo Ochoa SEV-2016-0597. The work of P.A.C. was supported by a "la Caixa-Severo Ochoa" International pre-doctoral grant. TO wishes to thank M.M. Fernández for her permanent support.

Open Access. This article is distributed under the terms of the Creative Commons Attribution License (CC-BY 4.0), which permits any use, distribution and reproduction in any medium, provided the original author(s) and source are credited.

\section{References}

[1] M. Hübscher, P. Meessen, T. Ortín and S. Vaulà, $N=2$ Einstein-Yang-Mills's BPS solutions, JHEP 09 (2008) 099 [arXiv: 0806.1477] [INSPIRE].

[2] M. Hübscher, P. Meessen, T. Ortín and S. Vaulà, Supersymmetric $N=2$ Einstein-Yang-Mills monopoles and covariant attractors, Phys. Rev. D 78 (2008) 065031 [arXiv:0712.1530] [INSPIRE].

[3] P. Meessen, Supersymmetric coloured/hairy black holes, Phys. Lett. B 665 (2008) 388 [arXiv: 0803.0684] [INSPIRE].

[4] P. Bueno, P. Meessen, T. Ortín and P.F. Ramírez, $\mathcal{N}=2$ Einstein-Yang-Mills' static two-center solutions, JHEP 12 (2014) 093 [arXiv:1410.4160] [INSPIRE].

[5] P. Meessen and T. Ortín, $\mathcal{N}=2$ super-EYM coloured black holes from defective Lax matrices, JHEP 04 (2015) 100 [arXiv: 1501.02078] [INSPIRE].

[6] P. Meessen, T. Ortín and P.F. Ramírez, Non-Abelian, supersymmetric black holes and strings in 5 dimensions, JHEP 03 (2016) 112 [arXiv: 1512.07131] [INSPIRE].

[7] T. Ortín and P.F. Ramírez, A non-Abelian Black Ring, Phys. Lett. B 760 (2016) 475 [arXiv: 1605.00005] [INSPIRE].

[8] P.A. Cano, P. Meessen, T. Ortín and P.F. Ramírez, Non-Abelian black holes in string theory, arXiv:1704.01134 [INSPIRE].

[9] A. Strominger, Heterotic solitons, Nucl. Phys. B 343 (1990) 167 [Erratum ibid. B 353 (1991) 565] [INSPIRE].

[10] P.A. Cano, T. Ortín and P.F. Ramírez, A gravitating Yang-Mills instanton, JHEP 07 (2017) 011 [arXiv: 1704.00504] [INSPIRE].

[11] P. Meessen, T. Ortín and P.F. Ramírez, Dyonic black holes at arbitrary locations, JHEP 10 (2017) 066 [arXiv:1707.03846] [INSPIRE].

[12] B. Bertotti, Uniform electromagnetic field in the theory of general relativity, Phys. Rev. 116 (1959) 1331 [INSPIRE].

[13] I. Robinson, A Solution of the Maxwell-Einstein Equations, Bull. Acad. Pol. Sci. Ser. Sci. Math. Astron. Phys. 7 (1959) 351 [inSPIRE]. 
[14] P.A. Cano, T. Ortín and C. Santoli, Non-Abelian black string solutions of $\mathcal{N}=(2,0), D=6$ supergravity, JHEP 12 (2016) 112 [arXiv:1607.02595] [INSPIRE].

[15] G.W. Gibbons and S.W. Hawking, Euclidean quantum gravity, World Scientific, Singapore, Singapore (1993).

[16] S.W. Hawking, G.T. Horowitz and S.F. Ross, Entropy, Area and black hole pairs, Phys. Rev. D 51 (1995) 4302 [gr-qc/9409013] [INSPIRE].

[17] G.W. Gibbons and R.E. Kallosh, Topology, entropy and Witten index of dilaton black holes, Phys. Rev. D 51 (1995) 2839 [hep-th/9407118] [InSPIRE].

[18] E.A. Bergshoeff and M. de Roo, The Quartic Effective Action of the Heterotic String and Supersymmetry, Nucl. Phys. B 328 (1989) 439 [InSPIRE].

[19] N. Halmagyi, D. Israel and E.E. Svanes, The Abelian Heterotic Conifold, JHEP 07 (2016) 029 [arXiv: 1601.07561] [INSPIRE].

[20] N. Halmagyi, D. Israel, M. Sarkis and E.E. Svanes, Heterotic Hyper-Kähler flux backgrounds, JHEP 08 (2017) 138 [arXiv: 1706.01725] [INSPIRE].

[21] G.W. Gibbons and S.W. Hawking, Action Integrals and Partition Functions in Quantum Gravity, Phys. Rev. D 15 (1977) 2752 [InSPIRE].

[22] M. Visser, How to Wick rotate generic curved spacetime, arXiv:1702.05572 [INSPIRE].

[23] A. Strominger and C. Vafa, Microscopic origin of the Bekenstein-Hawking entropy, Phys. Lett. B 379 (1996) 99 [hep-th/9601029] [INSPIRE].

[24] C.G. Callan and J.M. Maldacena, D-brane approach to black hole quantum mechanics, Nucl. Phys. B 472 (1996) 591 [hep-th/9602043] [INSPIRE].

[25] J.M. Maldacena, Black holes in string theory, Ph.D. Thesis, Princeton University (1996) [hep-th/9607235].

[26] R. Kallosh, T. Ortín and A.W. Peet, Entropy and action of dilaton black holes, Phys. Rev. D 47 (1993) 5400 [hep-th/9211015] [INSPIRE].

[27] D. Brill, Splitting of an extremal Reissner-Nordström throat via quantum tunneling, Phys. Rev. D 46 (1992) 1560 [hep-th/9202037] [INSPIRE].

[28] A. Papaetrou, A static solution of the equations of the gravitational field for an arbitrary charge distribution, Proc. Roy. Irish Acad. A 51 (1947) 191 [INSPIRE].

[29] S.D. Majumdar, A class of exact solutions of Einstein's field equations, Phys. Rev. 72 (1947) 390 [INSPIRE]. 